\title{
MIMO-OFDM signal optimization for SAR imaging radar
}

\author{
J.-Y. Baudais ${ }^{1 *}$, S. Méric ${ }^{2}$, V. Riché ${ }^{3}$ and É. Pottier ${ }^{3}$
}

\begin{abstract}
This paper investigates the optimization of the coded orthogonal frequency division multiplexing (OFDM) transmitted signal in a synthetic aperture radar (SAR) context. We propose to design OFDM signals to achieve range ambiguity mitigation. Indeed, range ambiguities are well known to be a limitation for SAR systems which operates with pulsed transmitted signal. The ambiguous reflected signal corresponding to one pulse is then detected when the radar has already transmitted the next pulse. In this paper, we demonstrate that the range ambiguity mitigation is possible by using orthogonal transmitted wave as OFDM pulses. The coded OFDM signal is optimized through genetic optimization procedures based on radar image quality parameters. Moreover, we propose to design a multiple-input multiple-output (MIMO) configuration to enhance the noise robustness of a radar system and this configuration is mainly efficient in the case of using orthogonal waves as OFDM pulses. The results we obtain show that OFDM signals outperform conventional radar chirps for range ambiguity suppression and for robustness enhancement in $2 \times 2 \mathrm{MIMO}$ configuration.
\end{abstract}

Keywords: Range ambiguity, SAR processing, OFDM signals, Image quality parameters, MIMO SAR, Genetic algorithm

\section{Introduction}

In radar domain, high resolution and good robustness against the noise are essential in detection and imaging processing. The high resolution is obtained with the synthetic aperture radar (SAR) principle which is largely detailed in the literature $[1,2]$. The robustness can be improved with multiple-input multiple-output (MIMO) configurations [3]. However, SAR images can be degraded with interferences that depend both on the radar scene and on the radar system.

One of the major interferences for the pulsed radar system is the arrival of unwanted echoes after the transmission of a new pulse. The range ambiguity then appears if the current transmitted pulse does not contribute to the back-scattered signal received after the transmission of this pulse. These late arrival echoes can appear when there are strong reflectors located after the maximum range detected by the radar system, namely ambiguity range. These kinds of echoes induce shadows in the image, which can be misinterpreted. Removal of this kind

*Correspondence: jean-yves.baudais@insa-rennes.fr

${ }^{1}$ IETR, UMR-CNRS 6164, CNRS, Rennes, France

Full list of author information is available at the end of the article of ambiguities is an important matter for SAR images. The fundamental and theoretical analysis on ambiguities have been already achieved [2]. The ambiguity treatment remains an important issue as the pulse repetition frequency (PRF) raises, whereas reasonable swath widths are required [4]. The use of a low PRF makes possible to mitigate range ambiguities but induces Doppler ambiguities degrading the azimuth resolution. To solve this trade-off, several ambiguity suppression techniques are proposed. For example, the use of alternating up and down chirp modulation is often presented [5]. Another technique is based on the azimuth phase coding to reduce the ambiguities [6]. Pulse block coding technique also makes possible to detect false targets due to the range ambiguity through matched filtering operations [7]. Orthogonal frequency division multiplexing (OFDM) signals are also proposed to reduce the cross-correlation between each transmitted pulse [8]. Moreover, these OFDM signals are designed to optimize the imaging quality parameters using genetic algorithms $[9,10]$.

The robustness of the imaging system is another limitation of the SAR system. Instead of increasing the power, multiple-input multiple-output (MIMO) configurations can be used to increase the robustness of the system [3]. 
However, these MIMO configurations lead to multiple waveform interferences. For example, in a $2 \times 2$ MIMO configuration using up and down chirps, the robustness is increased but the SAR images are affected: artifacts called ghost effects appear in azimuth which can be misinterpreted. As for range ambiguity, these ghost effects are due to the non-zero cross-correlation between the two transmitted signals.

In this paper, we propose to extend the optimization of the OFDM signal to the MIMO configuration. The range ambiguity reduction achieved by optimizing OFDM signal in a range ambiguity scenario is extended to the MIMO configuration to reduce the ghost effect. The image quality parameters are then improved using both OFDM-optimized signals and MIMO configurations.

The paper is organized as follows. Section 2 describes the ambiguity phenomenon and describes the SAR system we propose to reduce this ambiguity. The OFDM signal designed for ambiguity reduction is presented in Section 3 where experimental aspects as peak-to-average ratio (PAPR) are considered. The optimization of the OFDM signals to reduce the range ambiguity is developed in Section 4 with the genetic algorithm based on the image quality parameters. The results are presented in Section 5 . We show how the signals can be selected with the genetic algorithm to improve the range ambiguity suppression. In Section 6, the OFDM signals obtained in Section 4 are applied in the MIMO configuration and the imaging radar performances are evaluated. Finally, Section 7 concludes the paper.

\section{Range ambiguity}

The configuration shown in Fig. 1 is considered. The SAR system is a single-input single-output radar system characterized by its PRF and its swath width. The scene is divided in two parts: $(i)$ an unambiguous scene where all the reflectors located on are correctly imaged by the SAR system and (ii) an ambiguous scene within a single range ambiguous reflector. Let us consider a radar system where each pulse is coded with the same signal. The echoes corresponding to the $n$th transmitted pulse $s_{n}(t)$ and reflected by the ambiguous reflector arrives after the radar has already transmitted the $n+1$ th pulse $s_{n+1}(t)$, as shown in Fig. 2. This configuration yields to an ambiguity at time $\tau_{n+1}$ instead as unambiguous echo at time $\tau_{n}$. Regarding the radar characteristics given in Table 1, SAR processing is applied. As shown in Fig. 3, the reflector located at $(1150,0) \mathrm{m}$ is seen in the radar image around the $200 \mathrm{~m}$ range position which is considered as interference in this case. Moreover, defocusing phenomenon occurs along the azimuth axis.

Although the range ambiguity mainly appears with high wide swath in satellite configuration, we consider in this paper an airborne geometric configuration to compute the simulation and evaluate the performance of the proposed SAR imaging system in reasonable time. Thus, high value of the PRF is chosen to reinforce the ambiguity phenomena.

Now, let consider that two successive pulses are coded with two different waveforms. Let also consider $s_{a}(t)=$ $s_{n}(t)$ and $s_{b}(t)=s_{n+1}(t)$ be the two different signal waveforms used. The range ambiguity suppression is performed by applying multiple matched filters during the SAR processing. Let $s_{r(n+1)}(t)$ be the received signal composed of unambiguous echoes coming from $s_{b}(t)$ and composed of ambiguous echoes coming from $s_{a}(t)$. This signal $s_{r(n+1)}(t)$ is

$$
s_{r(n+1)}(t)=\sum_{i \in \mathcal{I}} s_{a}\left(t-\tau_{i}\right)+\sum_{i \in \mathcal{K}} s_{b}\left(t-\tau_{i}\right),
$$

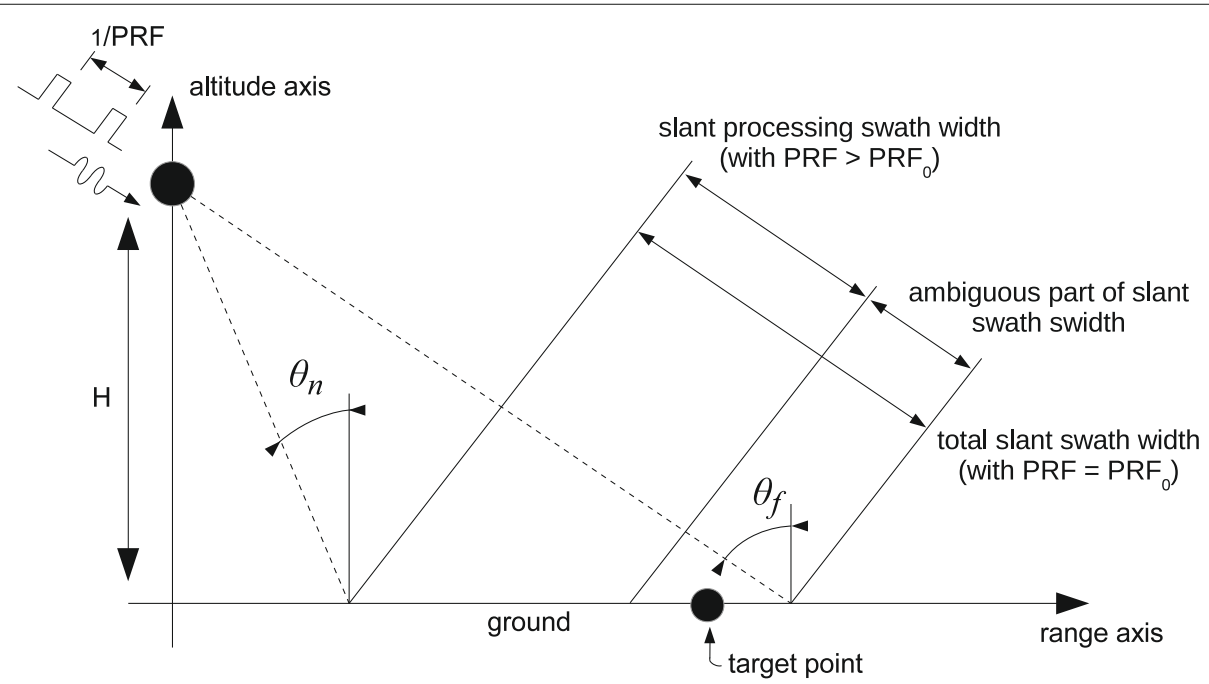

Fig. 1 SAR geometrical imaging configuration with range ambiguity 


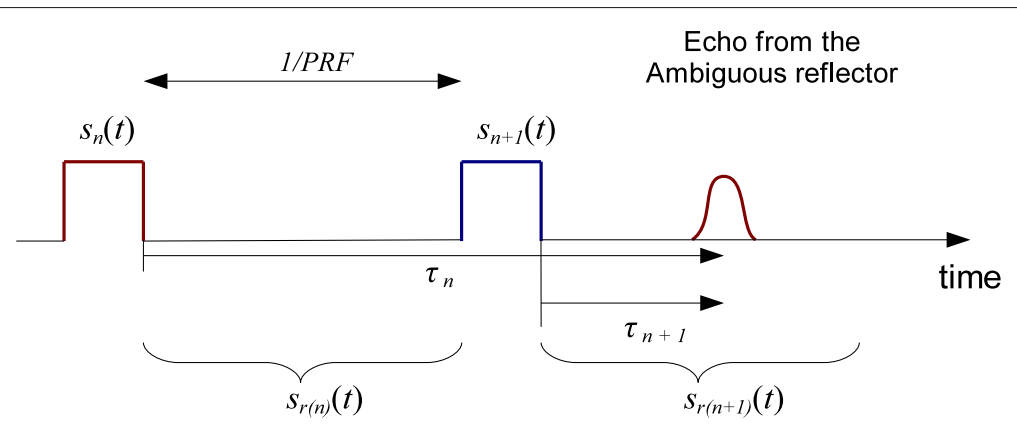

Fig. 2 Range ambiguity configuration

where $\tau_{i}$ is the delay related to the $i$ reflector, $\mathcal{I}$ is the set of ambiguous echoes and $\mathcal{K}$ is the set of unambiguous echoes. To mitigate the range ambiguity due to $s_{a}\left(t-\tau_{i}\right)$, the procedure described in Fig. 4 is applied. At the first step of this procedure, the filters matched to $s_{a}(t)$ and $s_{b}(t)$ are applied to the received signal. These range matched filters are followed by the azimuth matched filters and the Stolt interpolations. The matched filters in azimuth and the Stolt interpolations are chosen depending on whether they are applied either on the ambiguous case or the unambiguous case. The final step is to combine the two images obtained after each Stolt interpolation to obtain the complete image of the scene without any range ambiguities. The key point of this procedure is the ability of the two matched filters in range to reject the unmatched signals. Thus, the two transmitted signals $s_{a}(t)$ and $s_{b}(t)$ have to be chosen orthogonal or with low cross-correlation levels. Note that the ambiguity suppression procedure is presented in the case where the signal $s_{a}(t)$ and $s_{b}(t)$ are related to the index pulse $n$ and $n+1$ respectively. The role of $s_{a}(t)$ and $s_{b}(t)$ has to be switched for the processing of time pulses $n+1$ and $n+2$.

The use of the up and down chirp signals to reduce the cross-correlation has already been proposed [5]. In our radar configuration, the results obtained by these chirp signals are shown in Fig. 5. By using the range ambiguity suppression procedure previously described,

Table 1 SAR configuration

\begin{tabular}{ll}
\hline Parameters & Values \\
\hline $\mathrm{RF} f_{0}$ & $6 \mathrm{GHz}$ \\
Bandwidth $B$ & $20 \mathrm{MHz}$ \\
Pulse duration $T_{p}$ & $650 \mathrm{~ns}$ \\
PRF & $26.92 \mathrm{kHz}$ \\
Range ambiguity & $1 \mathrm{~km}$ \\
Range resolution $\delta_{r}$ & $7.5 \mathrm{~m}$ \\
Azimuth resolution $\delta_{\mathrm{az}}$ & $0.56 \mathrm{~m}$ \\
Ambiguous reflector position $(x, y)$ & $(1150,0) \mathrm{m}$ \\
\hline
\end{tabular}

the ambiguous reflector is now correctly located in range and also correctly focused in azimuth. However, a residual interference appears at the previous ambiguous position, which is called ghost effect. This ghost is due to the non orthogonality of the two chirps [11]. To reduce this ghost effect, we propose to use another waveform widely used in digital communication systems to address orthogonal multiple access: the OFDM signal [12].

\section{Design of OFDM signal}

\subsection{Signal definition}

We consider an OFDM signal $s_{a}(t)$ which is transmitted at a carrier frequency $f_{c}$ and constituted with $N$ sub-bands. To simplify the presentation, let us suppose that $N$ is even. Each sub-band has a $3 \mathrm{~dB}$-bandwidth $B_{i}$ and the total bandwidth of $s_{a}(t)$ is $B$. We define the baseband frequency $f_{i}$ as the sub-carrier frequency of the sub-band $i$. The emitted OFDM signal can be formulated as [13]

$$
s_{a}(t)=e^{j 2 \pi f_{c} t} \sum_{i=1}^{N} p(t) a_{i} e^{j 2 \pi f_{i} t},
$$

with $p(t)$ the pulse shaping function. The OFDM signal is coded by the vector a of coefficients $\left\{a_{i}\right\}_{i=1}^{N}$. The $i$ th coefficient corresponds to the activation of the sub-band $i$, $a_{i} \in\{0,1\}$, and $a_{i}=0$ means that the sub-band $i$ is turned off. Moreover, the baseband frequency $f_{i}$ of each sub-band is

$$
f_{i}=\frac{i-\frac{N-1}{2}-1}{T_{p}} .
$$

Finally, the function $p(t)=\operatorname{rect} \frac{t}{T_{p}}$ is the shaping function where $T_{p}$ is the OFDM symbol duration and $T_{p}^{-1}=f_{i+1}-$ $f_{i}$. The spectrum of each sub-band $i$ is a sinc-shape and centered at the sub-carrier frequency $f_{i}$.

\subsection{SAR application}

SAR OFDM signal has been studied for a decade $[8,14]$. To solve the range ambiguity, two signals exhibiting a low cross-correlation are used. Real baseband constraints have been used to design the OFDM signals in $[8,14]$. In our 


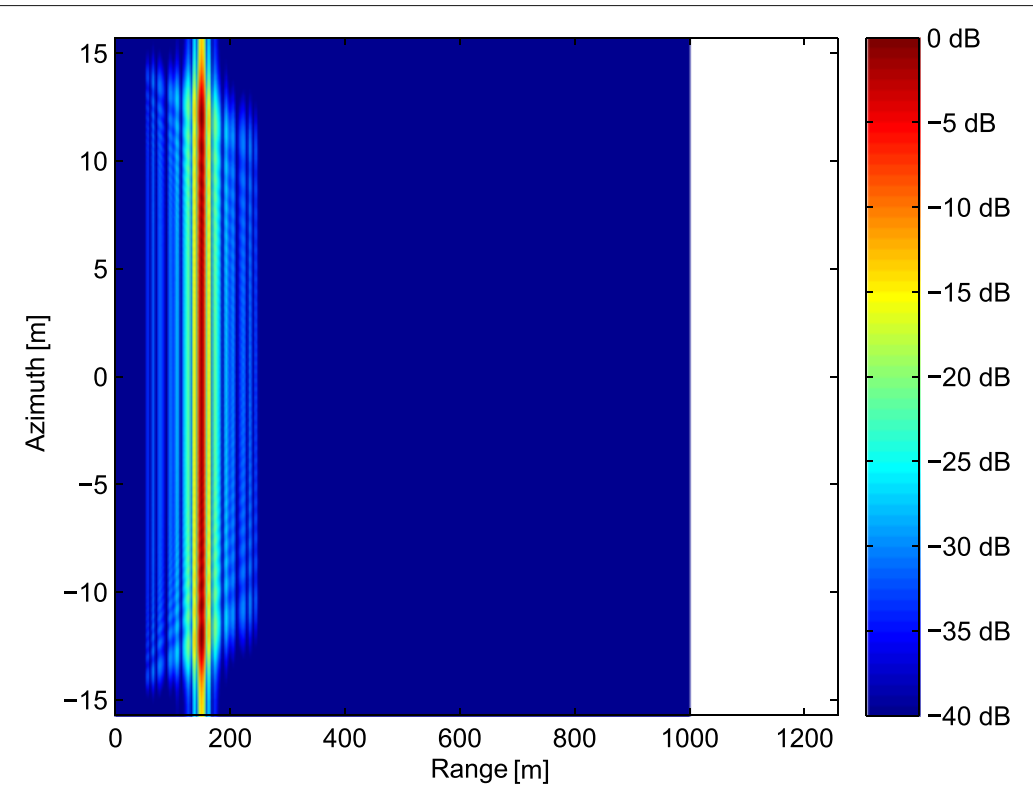

Fig. 3 SAR image with range ambiguity

study, we suppress this constraint to increase the design flexibility for these OFDM signals.

To simplify the SAR imaging signal processing, both signals $s_{a}$, and $s_{b}$, coded with the vector $\mathbf{a}$ and $\mathbf{b}$ respectively, must have the same carrier frequency, the same sub-carrier frequency $f_{i}$ and the same bandwidth. The orthogonality is then obtain by activating and turning off the different sub-bands of $s_{a}$ and $s_{b}$. This turning off introduces holes in the OFDM spectrum that could induce worse range resolution. However, these holes do not

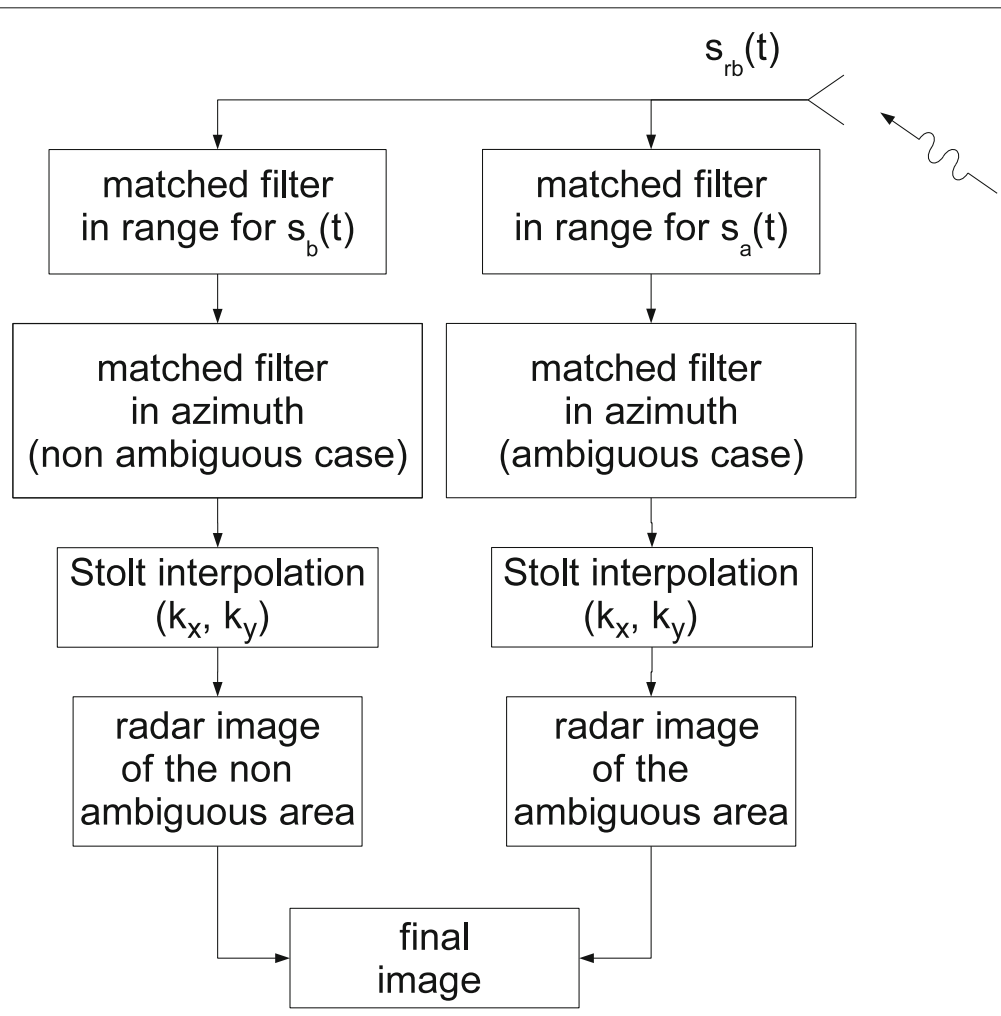

Fig. 4 Range ambiguity suppression procedure 


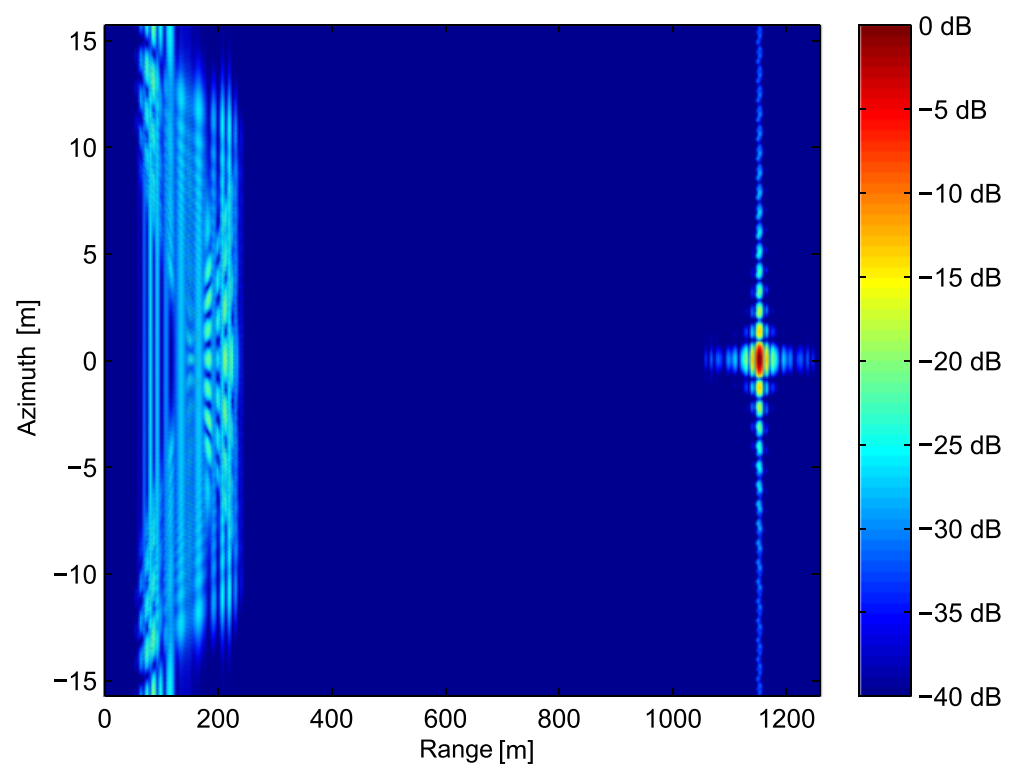

Fig. 5 SAR image with the range ambiguity suppression procedure using up and down chirp signal

significantly increase the range resolution value as shown in [9]. Consequently, we have to choose $\left(b_{i}\right)_{i=1}^{N}$ as the complementarity sequence of $\left(a_{i}\right)_{i=1}^{N}$ and the vector $\mathbf{b}$ can be define as $\mathbf{b}=\left[\bar{a}_{1}, \bar{a}_{2}, \cdots, \bar{a}_{N}\right]$ with $\bar{a}_{i}=1-a_{i}$. Also, the total bandwidth is divided in half between the two signals $s_{a}$ and $s_{b}$. The next step is to maximize the bandwidth of each signal $s_{a}$ and $s_{b}$ to provide the same and highest range resolution. Then, the vectors $\mathbf{a}$ and $\mathbf{b}$ must verify $\mathbf{a}=$ $\left[0,1, a_{3}, \cdots, a_{N-2}, 0,1\right]$ and $\mathbf{b}=\left[1,0, \bar{a}_{3}, \cdots, \bar{a}_{N-2}, 1,0\right]$. Note that the DC component, represented by the subcarrier $i=(N+1) / 2$, of the baseband signals is set to zero for $s_{a}$ and $s_{b}$, then $a_{(N+1) / 2}=b_{(N+1) / 2}=0$. By setting the value of $\left(a_{i}\right)_{i \in[3, N-3] \backslash\{(N+1) / 2\}}$, the different sub-bands are activated or turned off.

Taking into account the characteristics of the vector $\mathbf{a}$, we can define $N_{\text {couple }}$ couples $\{\mathbf{a}, \mathbf{b}\}$ as solution couple, which solve the range ambiguity problem, and

$$
N_{\text {couple }}=\frac{(N-5) !}{2\left(\frac{N-5}{2} !\right)^{2}} .
$$

Considering a limited number of sub-bands, all the possible couples can be computed and the one with the best ambiguity suppression is chosen as the best solution. As an example, 13 sub-bands gives 35 couples. Nevertheless, for a large number of sub-bands, the number of possible couples grows rapidly and the time needed to compute all the results becomes prohibitively high. This method of exhaustive search is not appropriate to find the best solution couple. Then, we propose to use a genetic algorithm to provide a good solution couple as closed as possible to the best solution couple $[15,16]$. In our case, the proposed genetic algorithm is based on multi-objective strategy.

\subsection{PAPR considerations}

The main drawback of the OFDM signal is the very high PAPR values that complicates the power amplifier design and increases the power consumption. This PAPR is defined by [17]

$$
\mathrm{PAPR}=\frac{\max _{t}\left|s_{a}(t)\right|^{2}}{\frac{1}{T_{p}} \int_{0}^{T_{p}}\left|s_{a}(t)\right|^{2} d t} .
$$

Using (2) and assuming that $p(t)$ is a rectangular pulse shaping function, the power of the signal $s_{a}(t)$ can be just upperbounded

$$
\left|s_{a}(t)\right|^{2}=\left|\sum_{i=1}^{N} a_{i} e^{2 \pi f f_{i} t}\right|^{2} \leq \sum_{i=1}^{N}\left|a_{i}\right|^{2} .
$$

In our case, the vector a is a $0-1$ vector. Then, the upper limit is reached when $t=0$. Moreover, the PAPR of $0-1$ vector is proportional to the number of 1-elements in the vector. As a is a $0-1$ vector, the PAPR is simply given by the number of active subcarriers

$$
\mathrm{PAPR}=\frac{N-1}{2} .
$$

Many solutions developed for wireless communication systems can be used to reduce this PAPR $[18,19]$. As a simple solution, we propose to use $a_{i}$ such as

$$
a_{i}=\left\{\begin{array}{l}
e^{\theta_{i}}, \theta_{i} \in[0,2 \pi) \text { for active sub-band, } \\
0 \text { otherwise }
\end{array}\right.
$$

that means $a_{i}=e^{j \theta_{i}}$ instead of $a_{i}=1$ for non empty value of $a_{i}$. Indeed, a signal $s_{a}(t)$ with random phases $\theta_{i}$ and with a huge number of sub-bands (around $10^{4}$ ) shows a PAPR 
reduction around $30 \mathrm{~dB}$ with respect to the PAPR value with $\theta_{i}=0$.

\section{Signal selection}

The genetic algorithm is based on objective functions to compare the different solution couples. We use two image quality parameters and two signal parameters to set four objective functions up.

\subsection{Parameters}

Two SAR image parameters are commonly used to assess the image quality: the so-called peak side lobe ratio (PSLR) and the integrated side lobe ratio (ISLR). The PSLR is [20]

$$
\text { PSLR }=\frac{\max _{n}\left|y_{n}\right|^{2}}{\left|y_{0}\right|^{2}}
$$

and the ISLR is

$$
\operatorname{ISLR}=\frac{\sum_{n}\left|y_{n}\right|^{2}}{\left|y_{0}\right|^{2}},
$$

where $\left|y_{n}\right|^{2}$ is the energy of each pixel of the image outside the main lobe and $\left|y_{0}\right|^{2}$ is the energy contained inside the main lobe.

The third parameter we propose to use is based on the correlation function:

$$
\Delta F_{0}=\frac{\max _{\tau}\left(\left|\chi_{a, b}(\tau, 0)\right|\right)}{\left|\chi_{a, a}(0,0)\right|},
$$

where $\chi_{a, b}\left(\tau, f_{d}\right)$ is the cross-ambiguity function [21], with respect to the delay $\tau$ and the frequency Doppler $f_{d}$, between $s_{a}(t)$ and $s_{b}(t)$, and, using (2), is defined by

$$
\begin{aligned}
\chi_{a, b}\left(\tau, f_{d}\right)= & \left(T_{p}-|\tau|\right) \sum_{i=1}^{N} \sum_{k=1}^{N} 2 a_{i} b_{k} \exp \left(\jmath \pi\left(i-k+f_{d} T_{p}\right)\right) \\
& \times \exp \left(\jmath \pi\left(i+k-N-1+f_{d} T_{p}\right) \frac{\tau}{T_{p}}\right) \\
& \times \operatorname{sinc}\left(2\left(i-k+f_{d} T_{p}\right)\left(1-\frac{|\tau|}{T_{p}}\right)\right) .
\end{aligned}
$$

Regarding (11) and (12), one can consider that $\Delta F_{0}$ evaluates the normalized correlation between the transmitted OFDM signals $s_{a}(t)$ and $s_{b}(t)$. The objective for the ambiguity suppression is then to obtain $\Delta F_{0}$ as lower as possible.

The last parameter is the PAPR value given by (5). The objective is also to reduce this value as much as possible.

\subsection{Genetic algorithm}

The main goal of the proposed genetic algorithm is to take into account multiple objective functions through a single fitness function. This genetic algorithm detailed in Fig. 6 is

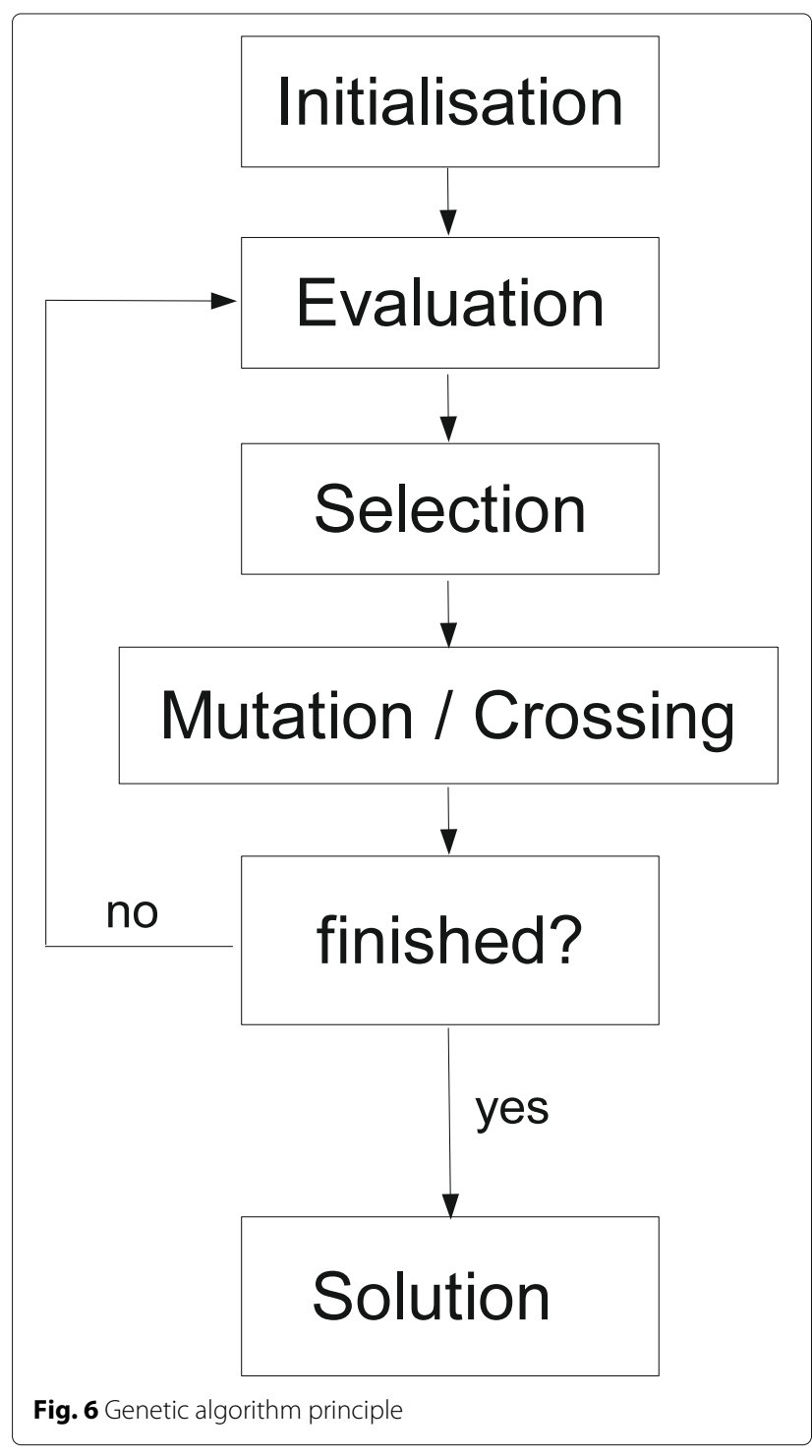

used to adjust the activation of the sub-bands in the vector a through three steps and one loop with ending condition.

Initialization: The first step initializes a population that contains the different vectors $\mathbf{a}$. This population of vectors is called the first generation.

Evaluation: In this step, the objective functions are evaluated. These objective functions $\left\{\Delta \phi_{i}(\mathbf{a})\right\}_{i=1}^{4}$ are the relative values of the PSLR for $i=1$, the ISLR for $i=2, \Delta F_{0}$ for $i=3$ and the PAPR for $i=4$. These objective functions are evaluated for each vector a of the population, which constitutes the current generation, and defined as

$$
\Delta \phi_{i}(\mathbf{a})=\frac{\left|\phi_{i}(\mathbf{a})-\phi_{i, 0}\right|}{\phi_{i, 0}}
$$

where $\phi_{i}(\mathbf{a})$ is the measured value of the ISLR, PSLR, $\Delta F_{0}$, or the PAPR for one given vector a. The term $\phi_{i, 0}$ 
is the reference value of the ISLR, PSLR, $\Delta F_{0}$, or the PAPR. For the PSLR and the ISLR, these reference values are obtained with a full-OFDM signal where all the sub-bands are activated (except the DC). For $\Delta F_{0}$, the reference value is an a priori value arbitrary chosen less than all possible $\phi_{3}(\mathbf{a})$. This value will be checked afterwards regarding the actual obtained solution couple. Concerning the PAPR, $\phi_{4,0}$ is the upper value given by (7), i.e., the maximal PAPR value for a vector a defined for SAR application in paragraph 3.2. Once the objective functions are defined, the fitness function $\psi$ is calculated using these objective functions and

$\psi(\mathbf{a})=\alpha_{1} \Delta \phi_{1}(\mathbf{a})+\alpha_{2} \Delta \phi_{2}(\mathbf{a})+\alpha_{3} \Delta \phi_{3}(\mathbf{a})+\alpha_{4} \Delta \phi_{4}(\mathbf{a})$,

where $\alpha_{i}$ is the weighting coefficient related to the objective function $\Delta \phi_{i}(\mathbf{a})$. As there is no priority objective function, these weighting coefficients are equal to 1 .

The value of the best fitness function of the current generation is defined as the lowest value of this fitness function, i.e. $\min _{\mathbf{a}} \psi(\mathbf{a})$. This value is then compared to the value of a reference fitness function, which is equal either to 1 at the beginning of the process or to the previous best fitness function. If the value of the current $\min _{\mathbf{a}} \psi(\mathbf{a})$ is lower than the value of the reference fitness function, the new reference fitness function becomes $\min _{\mathbf{a}} \psi(\mathbf{a})$. Otherwise, the reference fitness function remains unchanged.

Selection, mutation: After the evaluation, the selection and the mutation of the populations are processed. The chosen selection is a roulette-wheel selection with a $20 \%$ factor of mutation. It means that each element of the vectors a has a $20 \%$ chance to have its value changed (i.e., 0 becomes $e^{j \theta_{i}}$ and $e^{j \theta_{i}}$ becomes 0 ) between two successive generations. The factor of $20 \%$ is chosen to keep the search of a new solution close to the ones already found. By taking a higher factor, the research process tends to a random search.

The evaluation, selection, and mutation steps are included into a loop. As the aim of the genetic algorithm is not to search for the all possible solutions, the ending condition limits the searching sub-space dimension by defining the expected number of generations. In our approach, we limit this number of generations to 20 .

\section{Results with 65 sub-bands OFDM signal}

The under tested OFDM signal is composed of 65 subbands with a total bandwidth of $20 \mathrm{MHz}$. As explained in Section 3.2, half of these 65 sub-bands are activated and the other half are turned off. The number of possible couples, as defined in (4), is more than $59 \cdot 10^{15}$. The considered SAR configuration is the same as the one described in Section 2. As the pulse duration is inversely proportional to the bandwidth and proportional to the sub-band number, the pulse time duration becomes $T_{p}=$
Table 2 PSLR, ISLR and $\Delta F_{0}$ for the different OFDM-optimized solution couples

\begin{tabular}{llll}
\hline & PSLR $[\mathrm{dB}]$ & ISLR $[\mathrm{dB}]$ & $\Delta F_{0}[\mathrm{~dB}]$ \\
\hline$\left(s_{a, 1}, s_{b, 1}\right)$ & -18.5 & -43.6 & -11.3 \\
$\left(s_{a, 2}, s_{b, 2}\right)$ & -18.5 & -43.6 & -11.4 \\
$\left(s_{a, 3}, s_{b, 3}\right)$ & -18.5 & -43.5 & -11.6 \\
$\left(s_{a, 4}, s_{b}, 4\right)$ & -18.5 & -43.6 & -11.0 \\
$\left(s_{a, 5}, s_{b, 5}\right)$ & -18.3 & -43.4 & -12.1 \\
Full-OFDM & -16.4 & -48.1 & \\
Chirp couple & -13.9 & -40.8 & -9.2 \\
$\phi_{i, 0}$ & -20 & -48 & -14 \\
\hline
\end{tabular}

$\frac{65}{20 \cdot 10^{6}}=3.25 \mu \mathrm{s}$. Rising the number of sub-bands allows more flexibility for the OFDM signal design.

By using the genetic algorithm detailed in Section 4.2, we determine an optimized solution couple $\left(s_{a}, s_{b}\right)$. However, in this paper, the objective is to adjust and validate the activation of the sub-bands to mitigate the range ambiguity. We then reduce the research area and the complexity of the optimization problem by considering only $0-1$ coding vectors, i.e., $\theta_{i}=0$ in (8). We also consider the PAPR reduction as an independent task and do not address it in the following procedure.

In this paper, we focus on the sub-bands activation optimization. To validate the proposed approach, the genetic algorithm then uses only the three first objective functions and it is used five times with different initial populations. Five different optimized solution couples are then produced. The minimal reference values $\phi_{i, 0}$ used are given in Table 2. The fitness functions of the five optimized solutions are shown in Fig. 7. It can be seen that the algorithm converges from approximately the 15th generation.

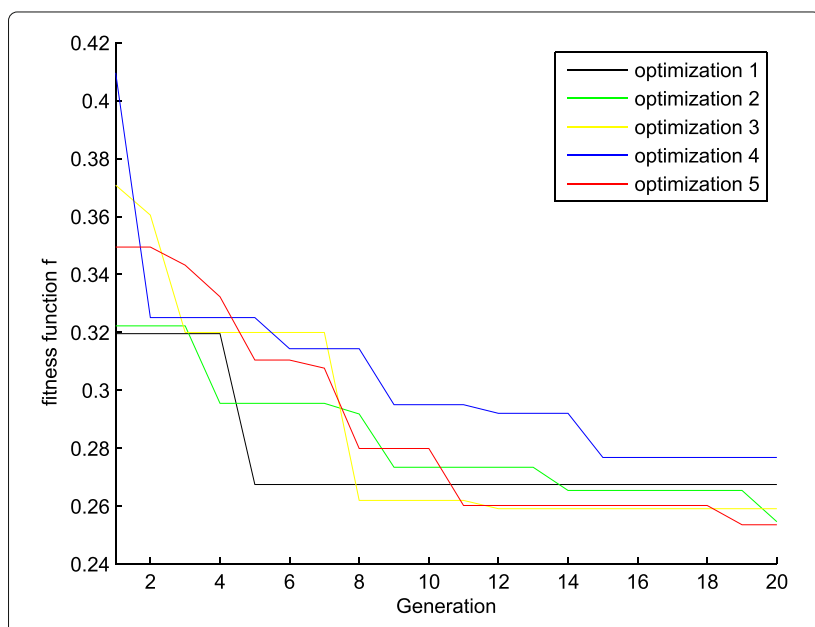

Fig. 7 Evolution of the fitness function 


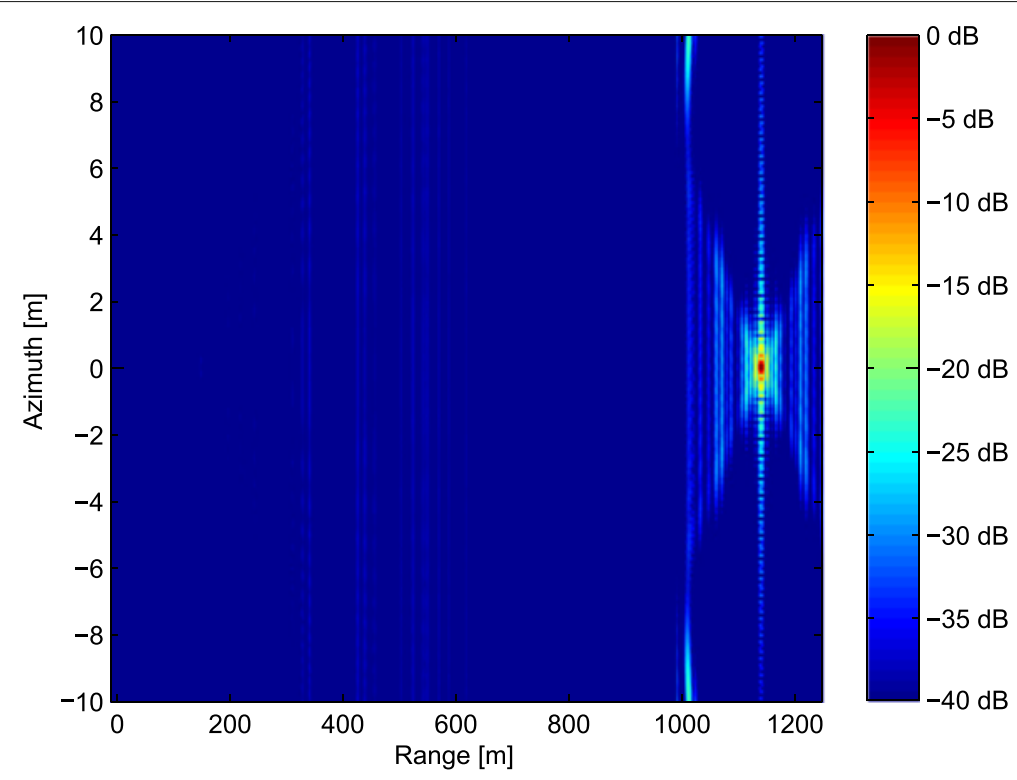

Fig. 8 SAR image with the range ambiguity suppression procedure using OFDM-optimized solution signals

To assess the advantages using optimized OFDM signals, the image quality parameters of the five optimized solution couples are compared to those obtained with the up and down chirps and also with the fullOFDM signal. The different values of the three parameters are shown in Table 2. Firstly, the image quality parameters of the five optimized OFDM signals are very closed each other. It means that the $20 \%$ mutation factor and the 20 generations are enough to ensure the convergence of the genetic algorithm. Secondly, all the OFDM solution couples provide better image quality parameters than the chirp couple does. Finally, the trade-off between the three SAR image quality parameters is achieved considering the expected reference values $\phi_{i, 0}$.

We can note that the ISLR values with optimized OFDM couples are larger than the full-OFDM signal one. The ISLR value differences are due to the holes in the spectra of the optimized OFDM signals. These holes induce an increase of the secondary lobes, which affect the value of the ISLR. Note also that $\Delta F_{0}$ value are not given for the full-OFDM signal because only one signal is generated in this case.

The SAR image shown in Fig. 8 is obtained with the first optimized couple $\left\{s_{a}, s_{b}\right\}$ which is noted $\left(s_{a, 1}, s_{b, 1}\right)$ in Table 2. This figure can be compared to Fig. 5 obtained in the case of up and down chirps. By analyzing these two figures, we notice that the ghost effect located around $150 \mathrm{~m}$ is much lower with the OFDM-optimized solution couples: in the chirps case, the level of the ghost is around $-35.7 \mathrm{~dB}$, whereas for the OFDM-optimized solution, this level is around $-46.7 \mathrm{~dB}$. The OFDM-optimized solution couple has then a better rejection of the ghost effect with a gain around $11 \mathrm{~dB}$.

\section{Extension to MIMO configuration}

The final objective in this paper is to improve the robustness of the imaging radar system using MIMO

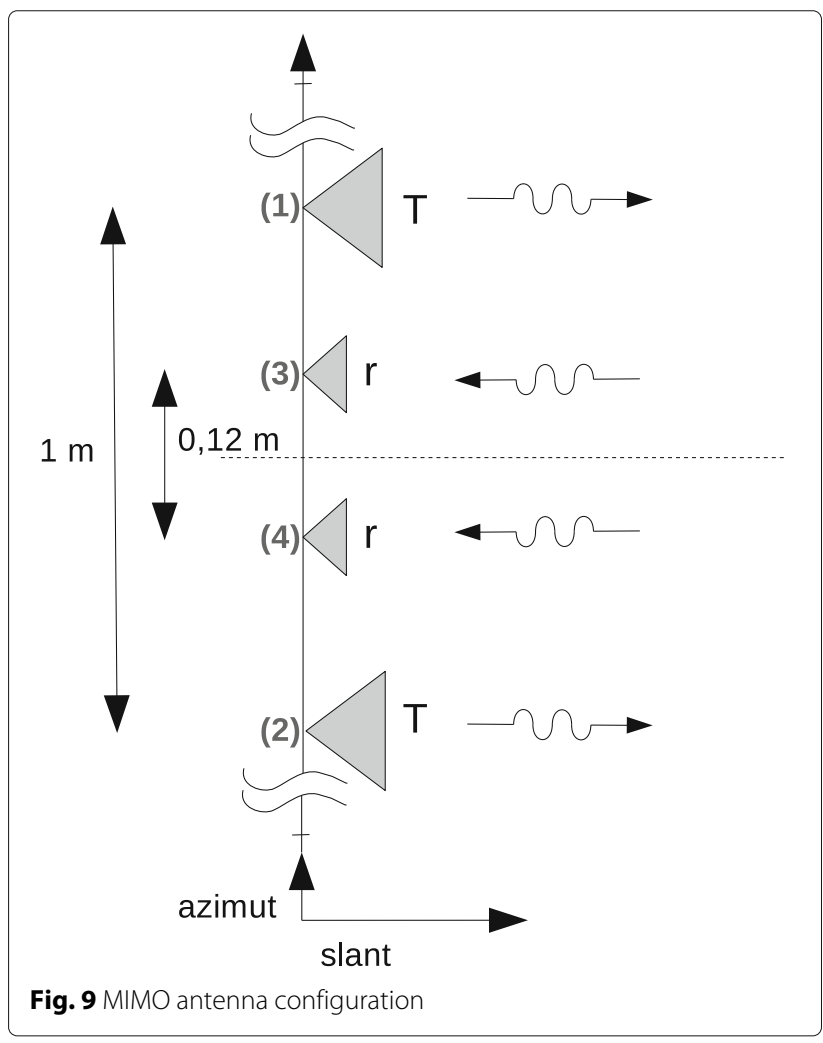




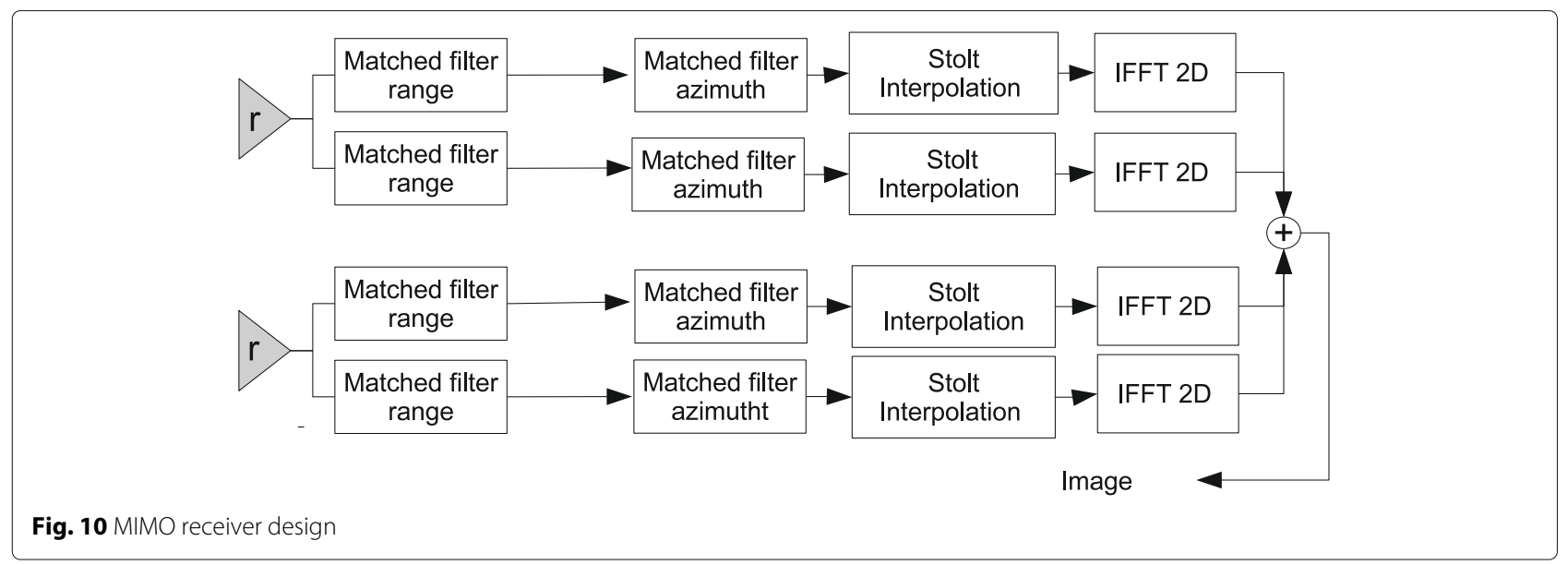

configuration without ambiguity case, as previously described. Multiple antennas lead to multiple signals emitted at the same time. Signals with low crossambiguity function are then needed to provide improvement. Firstly, we propose to use the optimized OFDM signals.

\subsection{MIMO configuration}

The MIMO configuration for SAR imaging is well described in the literature [22-24]. In this paper, we consider a MIMO SAR system composed of two transmitters and two receivers. The radar characteristics are given in Table 1, and the MIMO components of the system are shown in Fig. 9. The antennas are $12 \mathrm{~cm}$ apart at the receiver side and $1 \mathrm{~m}$ apart at the transmitter side. These distances correspond to $2 \times \lambda / 2$ and $42 \times \lambda / 2$, respectively. We consider a reflector positioned at the center of the observed scene, i.e., $0 \mathrm{~m}$ along the slant axis and $0 \mathrm{~m}$ along the azimuth axis.
The SAR imaging process is shown in Fig. 10 and corresponds to a basic coherent summation of the different images produced by each $\omega-\mathrm{k}$ processing [25]. The process to produce each image is the same as the one presented in Fig. 4. The coherent summation needs to focus in range and in azimuth each image and it takes into account the position of the different antennas to correctly coregister the different images.

\subsection{Simulation results}

Firstly, we propose two situations for each single-input single-output (SISO) and MIMO configurations. The first situation exhibits SAR image without additive noise and the second one exhibits image affected by an additive Gaussian noise (AWGN). This AWGN is designed to induce a $-30 \mathrm{~dB}$ level of SNR before the signal processing. Secondly, we propose to compare the SAR images achieved with

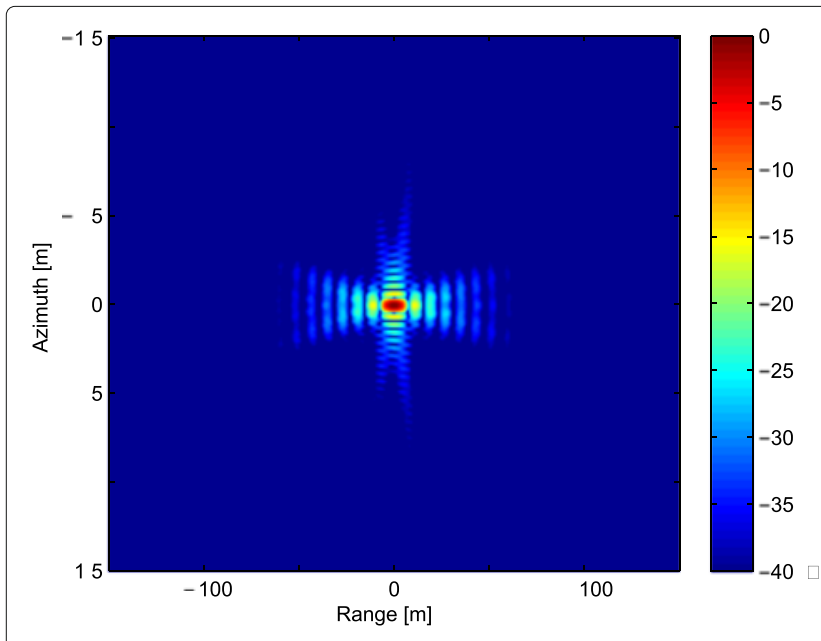

Without noise

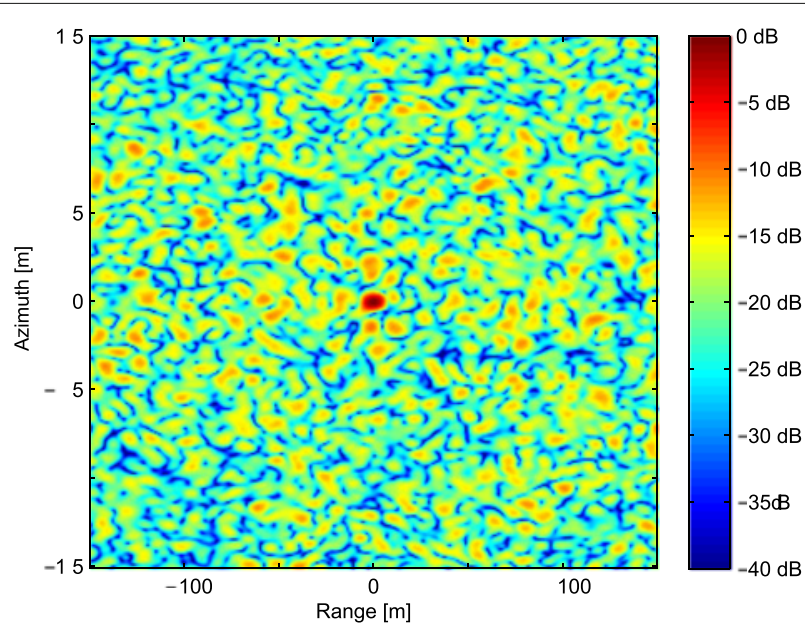

With noise

Fig. 11 SISO SAR image with chirp 


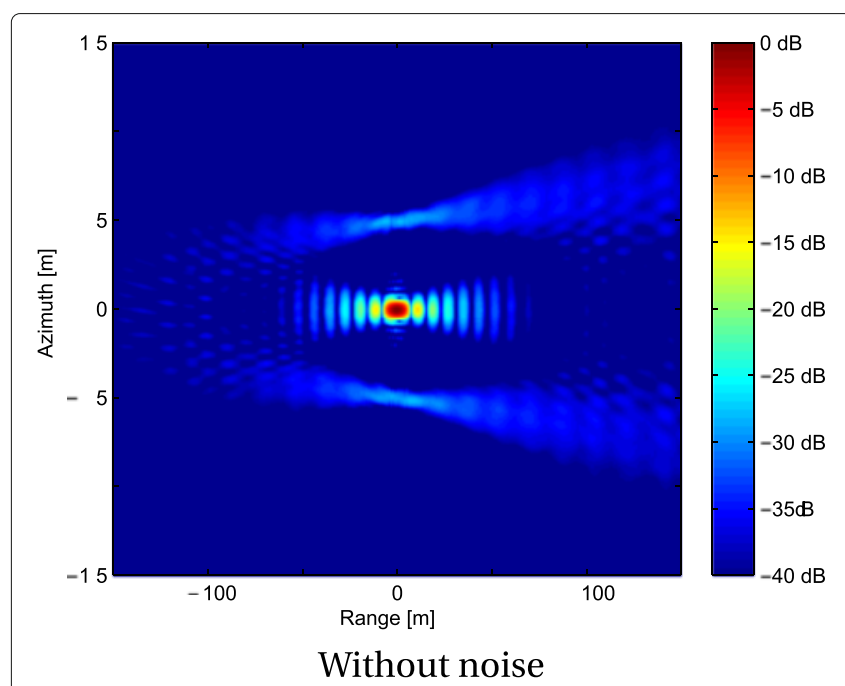

Fig. 12 MIMO SAR image with up and down chirps

1. a SISO configuration, Fig. 11,

2. a MIMO configuration using up and down chirps, Fig. 12,

3. a MIMO configuration using the OFDM signal optimized with respect to the image quality parameters, Fig. 13, as described in Section 4.

Figures 11, 12, and 13 describe the achieved SAR images that make possible to compare the different SAR configurations. Moreover, Table 3 qualitatively compares the image quality parameters that are measured in each configuration.

The results obtained with the up and down chirps (see Fig. 12) show some high side lobes around the coordinates $(x, y)=(0,5)$ in meter and $(0,-5)$. The maximum value of

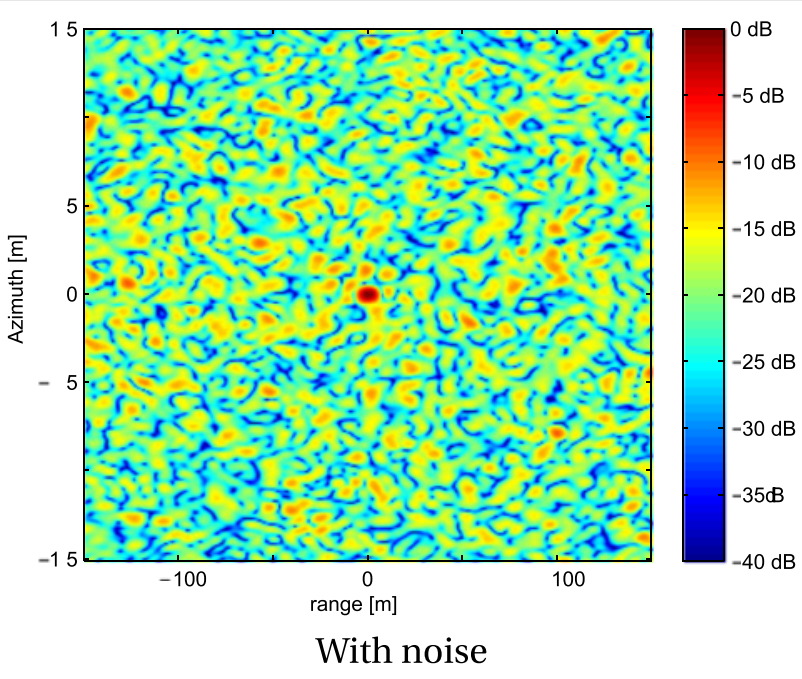

these side lobes is $27 \mathrm{~dB}$ lower than the main lobe. These two high side lobes have the same origin as the ghost in the range ambiguity configuration which is due to non orthogonality of the up and down chirps. As expected, the ghost effect is reduced with the MIMO OFDM signals, as shown in Fig. 13, with a gain higher than $5 \mathrm{~dB}$.

Table 3 compares the PSLR and ISLR values obtained with the different signals in the MIMO configuration. Both chirp and OFDM couples improve the parameters obtained in the SISO case. However, the use of the OFDMoptimized solution couple offers a PSLR gain of $0.7 \mathrm{~dB}$ and a ISLR gain of $1.4 \mathrm{~dB}$, comparing to the chirp couple. Note that the PSLR comparison does not measure the reduction of the ghost effect but only the level of the side lobe near the main lobe. Furthermore, the use of the OFDM signal

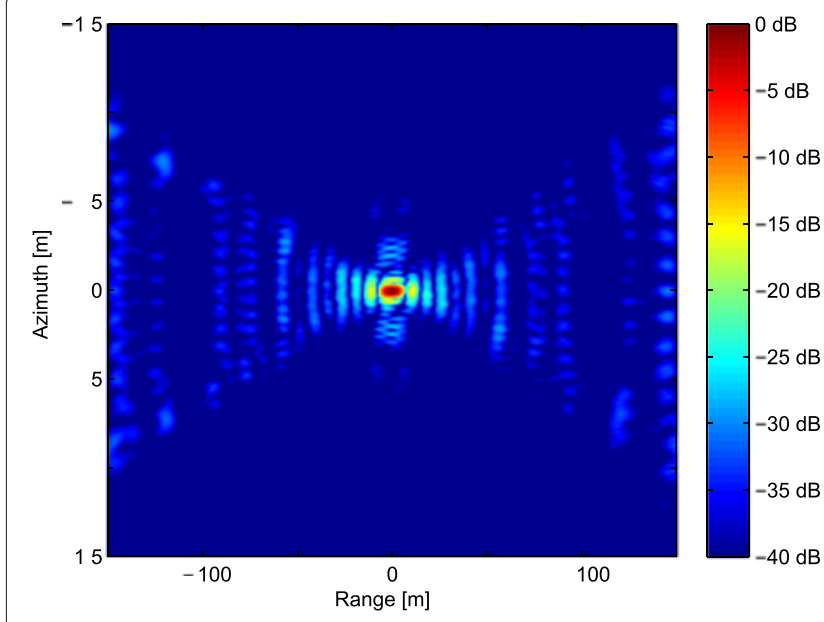

Without noise

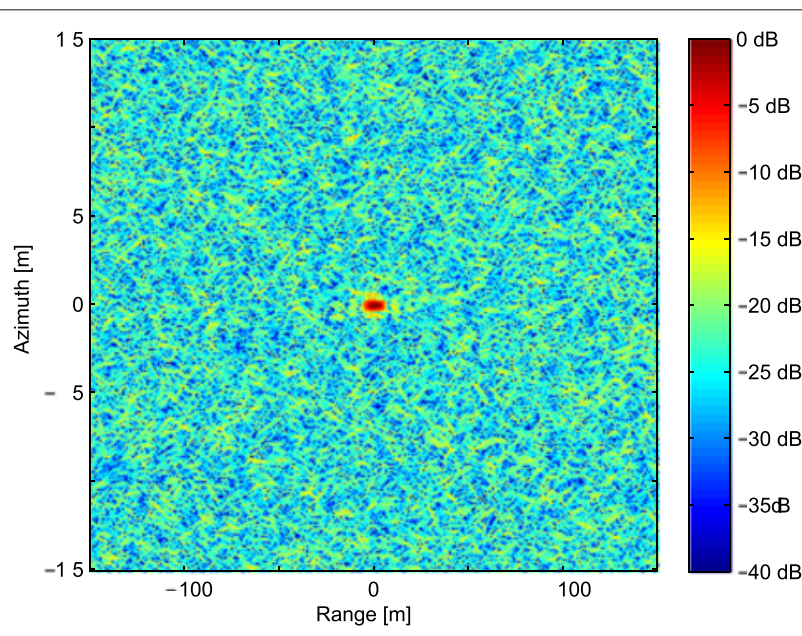

With noise

Fig. 13 MIMO SAR image using OFDM-optimized signals 
Table 3 Parameters of the SISO SAR, MIMO SAR, and MIMO OFDM SAR

\begin{tabular}{llll}
\hline Configuration & SISO & MIMO SAR & MIMO OFDM SAR \\
\hline$\delta_{r}[\mathrm{~m}]$ & 7.5 & 7.5 & 7.5 \\
$\delta_{\mathrm{az}}[\mathrm{m}]$ & 0.56 & 0.48 & 0.37 \\
PSLR [dB] & -13.8 & -14.2 & -14.9 \\
(with noise) & $(-9.5)$ & $(-11.2)$ & $(-12.0)$ \\
ISLR [dB] & -31.0 & -31.0 & -32.4 \\
(with noise) & $(-11.7)$ & $(-14.4)$ & $(-17.3)$ \\
\hline
\end{tabular}

has no influence in range, $\delta_{r}=7.5 \mathrm{~m}$, but offers a gain in azimuth of $34 \%$ with $\delta_{\mathrm{az}}=0.37 \mathrm{~m}$.

In the case of noisy SAR images, the value of the resolutions are unchanged. Nevertheless, the values of the other parameters are modified. By comparing the values of the different signals given in Table 3, a PSLR gain of $0.8 \mathrm{~dB}$ and an ISLR gain of $2.9 \mathrm{~dB}$ are achieved with the OFDM-optimized solution couple compared to the up and down chirps. It can be seen that while reducing the effect of the ghost, the use of OFDM-optimized signals, with the MIMO configuration, achieves a gain in the azimuth resolution and increases the robustness of the imaging system.

\section{Conclusions}

In this article, we demonstrate the possibility to mitigate the range ambiguity in a SAR image with OFDM signals and to reduce the ghost effect. A method based on the use of the genetic algorithm is proposed to optimize the signal and to reduce this ghost effect. The use of OFDMoptimized solution couple achieves a better rejection of the ghost effect compared to the use of the up and down chirp couple. The OFDM-optimized solution couple is applied to the MIMO configuration to also reduce the effect of the ghost appearing in azimuth. On the one hand, the use of the OFDM-optimized solution couple achieves a gain in the azimuth resolution and a gain of the PSLR and ISLR. On the other hand, a robustness gain of the imaging system is obtained with additive white Gaussian noise. However, one of the drawbacks of using the OFDM signals is the increase of the level of the side lobes in range due to the presence of holes in the transmitted signal spectrum. As we propose in the paper, further researches are turned into the optimization of the coded OFDM signal including the PAPR, which is one of the main drawbacks of OFDM signals in experimental systems.

\section{Competing interests}

The authors declare that they have no competing interests.

\section{Author details}

${ }^{1}$ IETR, UMR-CNRS 6164, CNRS, Rennes, France. ${ }^{2}$ IETR, UMR-CNRS 6164, INSA, Rennes, France. ${ }^{3}$ IETR, UMR-CNRS 6164, University of Rennes, Rennes, France.
Received: 29 April 2016 Accepted: 20 September 2016

Published online: 03 October 2016

\section{References}

1. M Soumekh, Synthetic Aperture Radar Signal Processing. (Wiley-Interscience, New-York, 1999)

2. I Cumming, F Wong, Digital processing of synthetic aperture radar data. (Artech House Publishers, ISBN: 978-1-58053-058-3, 2005)

3. J Li, P Stoica, X Zheng, Signal synthesis and receiver design for MIMO radar imaging. IEEE Trans. Signal Process. 56(8), 3959-3968 (2008)

4. J Mittermayer, M Younis, R Metzig, S Wollstadt, J Martínez, A Meta, TerraSAR-X system performance characterization and verification. IEEE Trans. Geosci. Remote Sensing. 48(2), 660-676 (2010)

5. J Mittermayer, J Martínez, in Internationnal Geoscience and Remote Sensing Symposium. Analysis of range ambiguity suppression in SAR by up and down chirp modulation for point and distributed targets (Toulouse, France, 2003), pp. 4077-4079

6. J Dall, A Kusk, in International Geoscience and Remote Sensing Symposium. Azimuth phase coding for range ambiguity suppression in SAR (Anchorage, Alaska, USA, 2004), pp. 4734-4737

7. J Akhtar, in Radar Conference. Cancellation of range ambiguities with block coding techniques (Pasadena, CA, USA, 2009), pp. 1-6

8. D Garmatyuk, in International Conference on Ultra-Wideband. Simulated imaging performance of UWB SAR based on OFDM (Waltham, MA, USA, 2006), pp. 237-242

9. V Riché, S Méric, J-Y Baudais, E Pottier, in European Radar Conference. Optimization of OFDM SAR signals for range ambiguity suppression (Amsterdam, Netherlands, 2012), pp. 278-281

10. W Mehany, L Jiao, X Zhang, Design discrete frequency coding waveform based OFDM for MIMO-SAR. Int. J. Inform. Electron. Eng. 5(2), 126-130 (2015)

11. U Stein, UM Younis, in International Conference on Computer as a Tool, vol 2. Suppression of range ambiguities in synthetic aperture radar systems (Faculty of Electrical Engineering, University of Ljubljana, Ljubljana, 2003), pp. 417-421

12. K Fazel, S Kaiser, Multi-carrier and spread spectrum techniques (From OFDM and MC-CDMA to LTE and WiMAX), 2nd ed. (John Wiley \& Sons Ltd, England, 2008)

13. G Franken, $H$ Nikookar, $P$ van Genderen, in European Radar Conference. Doppler tolerance of OFDM-coded radar signals (Manchester, United Kingdom, 2006), pp. 108-111

14. D Garmatyuk, M Brenneman, Adaptive multicarrier OFDM SAR signal processing. (Aachen, Germany, 2010), pp. 970-974

15. TMurata, $\mathrm{H}$ Ishibuchi, in International Conference on Evolutionary Computation, vol 1. MOGA: multi-objective genetic algorithms (University of Western Australia, Perth, Western Australia, 1995), p. 289

16. M Gen, R Cheng, Genetic algorithms and engineering optimization. (Wiley-Interscience, ISBN: 978-0471315315, 2000)

17. H Ochiai, $\mathrm{H}$ Imai, On the distribution of the peak-to-average power ratio in OFDM signals. IEEE Trans. Commun. 49(2), 282-289 (2001)

18. S Han, J Lee. An overview of peak-to-average power ratio reduction techniques for multicarrier transmission. IEEE Wireless Commun. 12(2), 56-65 (2005)

19. T Jiang, Y Wu, An overview: peak-to-average power ratio reduction techniques for ofdm signals. IEEE Trans. Broadcasting. 54(2), 257-268 (2008)

20. R Davis, R Fante, R Perry, Phase coded waveforms for radar. IEEE Trans. Aerospace Electron. Syst. 43(1), 401-408 (2007)

21. N Levanon, E Mozeson, Radar signals. ((Wiley-IEEE Press, New-Jersey, 2004)

22. J Klare, O Saalmann, H Wilden, A Brenner, in International Geoscience and Remote Sensing Symposium. Environmental monitoring with the imaging MIMO radars MIRA-CLE and MIRA-CLE X, (Honolulu, Hawaii, USA, 2010), pp. 3781-3784

23. J Klare, O Saalmann, H Wilden, A Brenner, in European Conference on Synthetic Aperture Radar. First experimental results with the imaging MIMO radar MIRA-CLE X (Aachen, Germany, 2010), pp. 1-4

24. J Ender, J Klare, in Radar Conference. System architectures and algorithms for radar imaging by MIMO-SAR, vol. 1 (Pasadena, CA, USA, 2009), pp. 1-6

25. SV Riché, E Méric, Pottier, Study of receiver design in a MIMO SAR configuration. Int. J. Microwave Wireless Technol. 4, 335-339 (2012) 